\title{
Improved FBG-Based Shape Sensing Methods for Vascular Catheterization Treatment
}

\author{
Omar Al-Ahmad ${ }^{1,2}$, Mouloud Ourak ${ }^{1}$, Jan Van Roosbroeck ${ }^{2}$, Johan Vlekken ${ }^{2}$ and Emmanuel Vander Poorten ${ }^{1}$
}

\begin{abstract}
Fiber optic shape sensing is gaining popularity within areas such as medical catheterization where catheters and guidewires are used to navigate through tortuous vascular paths. Shape sensing can aid medical interventionalists by reducing damaging radiation and providing a more detailed real-time understanding of the 3-dimensional shape of the catheter/guidewire. However, despite the technology existing for several years, there is still room for improvement and steps to follow to reach the accuracy and robustness needed for these safety-critical applications. This paper discusses and provides methods for fiber integration within catheters to improve shape estimation accuracy and repeatability. A two-step calibration process is introduced for intrinsic twist compensation, which results in significant improvements in estimation accuracy. Additionally, a practical method for fiber parameter identification is introduced. The importance of estimating these parameters was found to be paramount for reaching adequate shape estimation. Further improvements to the reconstruction algorithm are proposed. Experimental validations with ground truth shapes are performed to assess the overall accuracy for static and dynamic configurations. For complex geometrical shapes and a fiber length of $170 \mathrm{~mm}$, experiments show a mean spatial error of $0.70 \mathrm{~mm}(0.41 \%)$, a maximum of $2.52 \mathrm{~mm}(1.48 \%)$, and repeatability of $\pm 0.82 \mathrm{~mm}$.
\end{abstract}

\section{INTRODUCTION}

Recent technological advancements in fiber optic sensing allowed its use in various fields such as aerospace [1], [2] and medical treatment [3]-[5]. Fiber optic shape sensing (FOSS) made its first appearance in the mid 1980's where it was used to measure simple bends and curvatures [6], [7]. Since then, a large amount of work was done to improve sensor characteristics and performance. It was not until recent years that FOSS became very popular for various $2 \mathrm{D}$ and $3 \mathrm{D}$ shape sensing applications. Reasons for the increased interest in FOSS are mainly due to its advantages as a shape sensor, which include: 1) electromagnetic immunity, 2) miniature size, 3) high interrogation speed, 4) multiple sensing points, or multiplexing, 5) simple integration within systems, and 6) high mechanical strength [8], [9].

Several techniques exist with regards to fiber strain sensing. The two most common rely on Fiber Bragg Gratings (FBGs)

Manuscript received: January, 30, 2020; Revised March, 30, 2020; Accepted June, 7, 2020.

This paper was recommended for publication by Editor Pietro Valdastri upon evaluation of the Associate Editor and Reviewers' comments. This work was supported by the Flemish government VLAIO (HBC.2018.2046) and FBGS International.

${ }^{1}$ Omar Al-Ahmad, Mouloud Ourak and Emmanuel Vander Poorten are with the Department of Mechanical Engineering, KU Leuven University, Leuven, Belgium. (e-mail: firstname.lastname@kuleuven.be).

${ }^{2}$ Omar Al-Ahmad, Jan Van Roosbroeck and Johan Vlekken are with FBGS International NV, Geel, Belgium. (e-mail: oalahmad@fbgs.com, jvanroosbroeck@fbgs.com; jvlekken@fbgs.com).

Digital Object Identifier (DOI): see top of this page. and Rayleigh scattering. FBG-based strain sensing relies on reflected wavelengths from an emitted light spectrum. The reflected light depends on the grating periodicity which on its turn is function of the internal strain. The technology normally offers a discrete distribution of FBGs which result in strain measurements at a large number of discrete locations. Rayleigh scattering based techniques have near-continuous spatial strain sensing resolution. This is because scattering occurs throughout the fiber length. Optical Frequency Domain Reflectometry (OFDR) is the main interrogation technology used in conjunction with Rayleigh scattering based techniques. While OFDR provides for high spatial resolution and does not require FBG inscription, FBG-Based interrogation techniques such as Wavelength Division Multiplexing (WDM), which are spectrometry based technologies, are comparatively cheaper alternatives, typically have higher signal to noise (SNR) ratios [10], and higher measurement speeds.

Previous work has employed both FBG and Rayleigh scattering shape sensing techniques for various applications such as needle insertion and tracking [11]-[13], tracking of continuum manipulators [14]-[16], or generic tracking and localization applications [17]-[19]. Most previous approaches adopt a strategy where three outer single core fibers are used for shape reconstruction. This usually requires further calibration steps making it harder to reach the same level of precision as with a single multi-core fiber (MCF). The most commonly used reconstruction methods used in these works are based on Frenet-Serret frames, parallel transport or Bishop frames, constant curvature segmentation, and helical geometry. While most of these works show adequate shape accuracy for their given application, there is yet a large opportunity for improvement. This is especially the case for longer length fibers and environments with high curvature and torsion. In cardiovascular medical applications, the required accuracy that clinicians indicate as being acceptable is typically in the order of 1-3 mm [20]-[22], but may be lower for intricate interventions. Table I summarizes prior works on this subject and the corresponding reported errors.

The reconstruction accuracy of shape sensing schemes generally depends on factors such as:

- interrogation method: important factors include spatial resolution, repeatability, linearity, and sensitivity.

- fiber integration: crucial to ensure shape repeatability, avoid backscattering of light, avoid play due to extra spacing, and prevent twist.

- calibration and parameter identification: an important step that must be performed precisely to measure initial twist, and identify model parameters.

- twist compensation: initially constrained to remain con- 
TABLE I

SHAPE SENSING EXAMPLES FROM PREVIOUS LITERATURE (ALL DIMENSIONS ARE IN MILLIMETRE)

\begin{tabular}{|c|c|c|c|c|c|c|c|c|c|c|}
\hline Authors & Interrogation & Fibers \& Cores & Conf. & Model & Length & \#Grts & Spacing & Validation & Error & $\mathrm{R}_{\min }$ \\
\hline Abayazid et al. [11] & FBG / WDM & 3 outer / no central & Straight & Constant curvature & 90 & 4 & 30 & $2 \mathrm{D} / 3 \mathrm{D}$ & $2.10 \pm 1.10$ (mean) & 375 \\
\hline Henken et al. [12] & FBG / WDM & 3 outer / no central & Straight & Frenet-Serret & 70 & 2 & 70 & 2D & $1.32 \pm 0.48$ (mean) & NA \\
\hline Yi et al. [23] & FBG / WDM & 4 outer / no central & Straight & Frenet-Serret & 400 & 5 & 100 & $2 \mathrm{D} / 3 \mathrm{D}$ & 4.10 (mean) & NA \\
\hline Elayaperumal et al. [24] & FBG / WDM & 3 outer / no central & Straight & Other & 85 & 2 & 85 & $2 \mathrm{D}$ & $4.20(\mathrm{rms})$ & NA \\
\hline Gander et al. [25] & FBG / WDM & $4(\mathrm{MCF}) /$ no central & Straight & Other & NA & NA & NA & $2 \mathrm{D}$ & $2.00(\max )$ & 20 \\
\hline Van de Berg et al. [13] & FBG / WDM & 3 outer / no central & Straight & Frenet-Serret & 120 & 4 & 40 & $3 \mathrm{D}$ & $2.60 \pm 1.10($ mean $)$ & 71.4 \\
\hline Parent et al. [26] & OFDR & 3 outer / no central & Straight & Other & NA & NA & NA & $2 \mathrm{D}$ & $\approx 1.00(\mathrm{rms})$ & 17.5 \\
\hline Sefati et al. [14] & FBG / WDM & 3 outer / no central & Straight & Constant curvature & NA & NA & NA & $3 \mathrm{D}$ & $0.62(\max )$ & 101.6 \\
\hline Ryu et al. [17] & FBG / WDM & 3 outer / no central & Straight & Other & NA & NA & NA & $2 \mathrm{D}$ & $0.84 \pm 0.62($ mean $)$ & NA \\
\hline Leyendecker et al. [27] & FBG / WDM & $3(\mathrm{MCF}) /$ central & Straight & Constant curvature & 250 & 6 & 50 & $2 \mathrm{D} / 3 \mathrm{D}$ & $15.40(\max )$ & NA \\
\hline Lally et al. [28] & OFDR & 3 (MCF) / central & Helical & Other & 30000 & NA & NA & $3 \mathrm{D}$ & $210(\max )$ & NA \\
\hline Klute et al. [1] & OFDR & 3 outer / no central & Straight & Constant curvature & 2000 & NA & NA & $3 \mathrm{D}$ & $42.9(\max )$ & 14 \\
\hline Duncan et al. [10] & OFDR & 3 (MCF) / no central & Straight & NA & 1100 & 110 & 10 & $2 \mathrm{D}$ & $22.50 \pm 0.5(\max )$ & 667 \\
\hline Khan et al. [29] & FBG / WDM & $4(\mathrm{MCF}) /$ no central & Straight & Frenet-Serret & 108 & 6 & 18 & $2 \mathrm{D} / 3 \mathrm{D}$ & $1.05(\max )$ & NA \\
\hline Roesthuis et al. [18] & FBG / WDM & 3 outer / no central & Straight & Frenet-Serret & 90 & 4 & 3 & $2 \mathrm{D}$ & 1.14 (mean) & 30 \\
\hline Kim et al. [15] & FBG / WDM & 3 outer / no central & Straight & Constant curvature & 150 & NA & NA & $3 \mathrm{D}$ & $0.53(\max )$ & NA \\
\hline Wang et al. [30] & FBG / WDM & 4 outer / no central & Straight & Frenet-Serret & 200 & 5 & 50 & $3 \mathrm{D}$ & 15.00 (mean) & NA \\
\hline Moore et al. [2] & FBG / OFDR & $3(\mathrm{MCF}) /$ no central & Straight & Frenet-Serret & 1100 & 111 & 10 & $3 \mathrm{D}$ & $31.06(\max )$ & 14.3 \\
\hline Roesthuis et al. [4] & FBG / WDM & 3 outer / no central & Straight & Constant curvature & 90 & 4 & 30 & $2 \mathrm{D} / 3 \mathrm{D}$ & $1.66(\max )$ & 15 \\
\hline
\end{tabular}

stant, and then compensated (in general, shape sensors cannot measure twist independently).

- reconstruction algorithm: optimization of the reconstruction algorithm to take additional effects into account (e.g. reconstruction method, interpolation, etc.).

All of the previously outlined factors are discussed in this paper. Different ways to estimate and compensate for their effects are proposed and validated. This paper provides the following contributions: 1) an approach for fiber integration into a catheter that assures improved performance and robustness, 2) a two-step calibration method to measure intrinsic twist and compensate for it, 3) the use of a new approach for spatial curve reconstruction based on the Helical Extension Method (HEM) [31], 4) a method for parameter identification based on trust-region optimization, and 5) validations on diverse 2D and 3D shapes for static and dynamic configurations with ground truth data. The work in this paper focuses mainly on multicore fibers. However, it can be readily extended to a plurality of single core fibers having similar geometrical configurations.

This paper is structured as follows: Section II covers the basic principles of FBG strain sensing and the algorithm used for shape reconstruction. Section III discusses the main factors that contribute to shape accuracy, and proposes mitigating solutions. The experimental setup and methods are elaborated in Section IV, while Section V discusses the experimental results. Finally Section VI gives some concluding remarks and possibilities for future work.

\section{BASIC PRINCIPLES AND RECONSTRUCTION ALGORITHM}

The main principle behind shape reconstruction with FBGs is strain sensing. This is because the strain $\epsilon$ can be used to find the curvature $\kappa$ based on the well-known relation:

$$
\epsilon=-\kappa y,
$$

where $y$ is the distance from the neutral bending plane. This is of course assuming symmetry about the bending plane, no twisting (warping) and no buckling. FBGs detect variation of strain based on the change of periodicity and refractive index
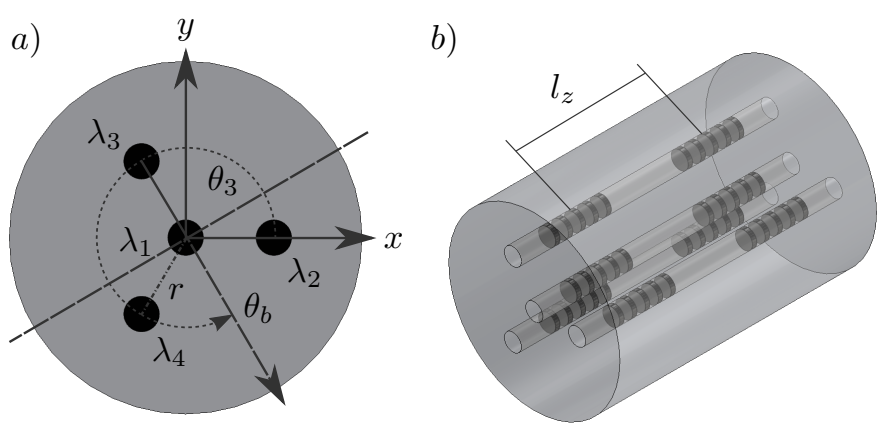

c)

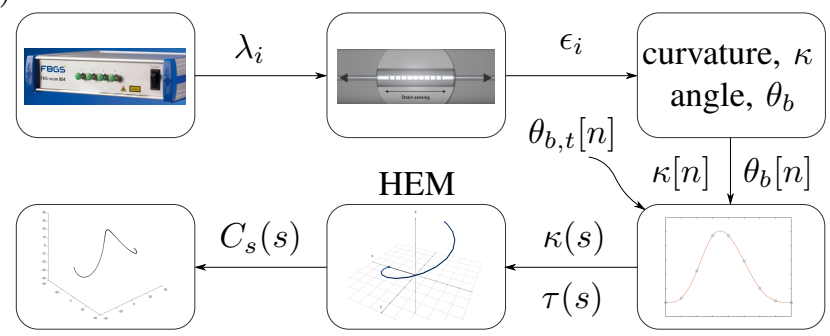

Fig. 1. a) cross-sectional view of a 4 core MCF where $\lambda_{i}$ represents the wavelength in the $i^{\text {th }}$ core, $\theta_{i}$ is the angle between the $x$-axis and the $i^{\text {th }}$ core measured counter-clockwise, $r$ is the distance between the center of the $i^{t h}$ core and the fiber's central axis, and $\theta_{b}$ is the angle of the bending plane with respect to the $x$-axis, b) isometric view of a short segment with four cores and two FBG sets separated by a grating center-to-center distance of $l_{z}$, c) reconstruction algorithm flowchart.

of the grating. The Bragg wavelength $\lambda_{B}$ is the wavelength of the light that is reflected back from the grating. The change in strain can be a mechanical strain $\epsilon$, or thermal expansion due to a temperature change $\Delta T$. Considering small temperature shifts, the change in Bragg wavelength can be expressed as:

$$
\frac{\lambda_{B}-\lambda_{B_{0}}}{\lambda_{B_{0}}}=\frac{\Delta \lambda_{B}}{\lambda_{B_{0}}}=S_{\epsilon} \Delta \epsilon+S_{T} \Delta T,
$$

where $\lambda_{B_{0}}$ is the grating's unstrained Bragg wavelength, $\Delta \epsilon$ is the change in strain with respect to the unstrained state, and $S_{\epsilon}$ and $S_{T}$ are the strain and temperature sensitivity coefficients, respectively. Multi-core fibers (MCFs) normally contain a central core that coincides with the fiber's neutral axis, in addition to a number of symmetrically positioned outer 
cores (see Fig. 1). Gratings located within the central core are only sensitive to strain due to axial loading and temperature fluctuations; they are not sensitive to bending as the neutral axis will not be affected by it. If axial strain is negligible, then the change in wavelength due to temperature change can be simply known by measuring the wavelength shift in the central core $\left(\Delta \lambda_{B, 1}\right)$. For the outer cores, the temperature contribution $S_{T} \Delta T$ is thus known from the central core, and (2) can be rewritten as:

$$
\Delta \epsilon_{i}=\frac{\Delta \lambda_{B, i}}{S_{\epsilon} \lambda_{B_{0}, i}}-\frac{\Delta \lambda_{B, 1}}{S_{\epsilon} \lambda_{B_{0}, 1}} .
$$

\section{A. Strain, Curvature and Bend Angle}

If twist and axial strain are assumed to be negligible, at least two unique strain measurements at different locations along the fiber's cross-section are required to determine the curvature and its direction (direction of the bending plane $\theta_{b}$ ). The relationship between the the strain in each core $\epsilon_{i}$ and the corresponding curvature $\kappa$ can be obtained from the geometry as depicted in Fig. 1:

$$
\epsilon_{i}=-\kappa r \sin \left(\theta_{b}-3 \pi / 2-\theta_{i}\right)
$$

where $r$ is the distance of the outer cores to the central core assuming a symmetrical configuration (i.e. $r$ is equal for all outer cores), and $\theta_{i}$ is the angle of the $i^{t h}$ core with respect to the $\mathrm{X}$-axis as shown in Fig. 1. As can be seen from (4), the two unknowns are curvature $\kappa$ and bend angle $\theta_{b}$. Hence only two outer core strain measurements are needed to solve for them. However, additional cores can be used to improve the result and reduce errors. A closed-form solution can be obtained for the curvature $\kappa$ and the bend angle $\theta_{b}$ by defining an apparent curvature vector $\kappa_{a p p}$ [32]:

$$
\begin{gathered}
\boldsymbol{\kappa}_{a p p}=-\sum_{i=1}^{N} \frac{\epsilon_{i}}{r} \cos \theta_{i} \hat{\boldsymbol{i}}-\sum_{i=1}^{N} \frac{\epsilon_{i}}{r} \sin \theta_{i} \hat{\boldsymbol{j}}, \\
\kappa=\frac{2\left|\boldsymbol{\kappa}_{a p p}\right|}{N}, \\
\theta_{b}=L \boldsymbol{\kappa}_{a p p},
\end{gathered}
$$

where $\hat{i}$ and $\hat{j}$ are the unit vectors along the $x$ - and $y$-axes respectively, $N$ is the number of outer cores, and $\angle$ represents the vector angle. The bend angle $\theta_{b}$ in (7) is obtained using the atan 2 function. This is used to limit the angle to the bounded interval $[-\pi \pi]$. If a MCF contains $n$ FBGs located at discrete intervals along its axis, (6) and (7) have to be computed $n$ times. This results in a set of curvatures $\kappa[n]$ and a set of bend angles $\theta_{b}[n]$ along the fiber's length.

\section{B. Shape Reconstruction}

Shape reconstruction begins from a set of measurements of the discrete FBG wavelengths $\lambda[n]$. A vector of reference wavelengths $\lambda_{0}[n]$ must be obtained a priori (for all sensor sets), and represents the unstrained Bragg wavelengths, i.e. $\lambda_{0}[n]=\lambda_{B_{0}}[n]$. The common mode is obtained by reading the wavelength of the central core $\lambda_{c}[n]$, which is consequently subtracted from $\lambda[n]$ and $\lambda_{0}[n]$. Hence, the compensated wavelengths are $\lambda_{\text {comp }}[n]=\lambda[n]-\lambda_{c}[n]$, and $\lambda_{c o m p, 0}[n]=$ $\lambda_{0}[n]-\lambda_{0, c}[n]$. The strains in the outer cores $\epsilon_{i}[n]$ are obtained by replacing $\lambda_{c o m p}[n]$ and $\lambda_{c o m p, 0}[n]$ into (3). The apparent curvature vector $\boldsymbol{\kappa}_{a p p}[n]$ can then be obtained by substituting $\epsilon_{i}[n]$ into (5). Finally, the curvatures $\kappa[n]$ and bend angles $\theta_{b}[n]$ are computed through (6) and (7), respectively.

A continuous and differentiable space curve can be defined by its curvature $\kappa(s)$ and torsion $\tau(s)$ profiles, where $s$ is the arc length variable. The torsion $\tau(s)$ is defined as the rate of change of the bend angle $\theta_{b}$ with respect to the arc length, i.e. $\tau(s)=\frac{d \theta_{b}}{d s}$. The curvature and torsion profiles define how the curve's tangent $\boldsymbol{T}$, normal $\boldsymbol{N}$, and binormal $\boldsymbol{B}$ unit vectors evolve along its length. This is mathematically defined using the differential Frenet-Serret formulae [33]. The differences in bend angles $\theta_{b}[n]$ are computed to obtain $\Delta \theta_{b}[n-1]$, which is wrapped in the bound $[-\pi \pi]$ to give a sense of rotation. To find the $n^{\text {th }}$ bend angle difference, $\Delta \theta_{b}[n-1]$ is extrapolated to obtain $\Delta \theta_{b}[n]$, which is finally divided by $l_{z}$ to obtain $\tau[n]$; where $l_{z}$ is the distance between consecutive FBGs. The discrete curvatures and torsions are then interpolated in order to improve shape estimation and obtain a quasi-continuous distribution along the fiber's arc length $s, \kappa[n] \rightarrow \kappa(s)$ and $\tau[n] \rightarrow \tau(s)$. The continuity of the interpolation is only guaranteed by the method used. Finally, the differential FrenetSerret formulae are solved to obtain the evolution of the TNB frame. The Cartesian position $C_{s}(s)$ can be obtained by integrating:

$$
C_{s}(s)=C_{s, 0}+\int_{0}^{l} \boldsymbol{T}(s) d s,
$$

where $C_{s, 0}$ is the Cartesian coordinate of the curve's base and $l$ is the interrogated length. A variety of methods can be used to solve (8). This will be further discussed in Section III where HEM is introduced. Figure 1(c) illustrates a summary flowchart of the reconstruction algorithm.

\section{CONTRIBUTORS TO SHAPE ACCURACY}

A plurality of factors, discussed next, affect the reconstructed shape accuracy.

\section{A. Interrogation Method}

WDM and OFDR are the most popular interrogation methods used nowadays. OFDR can be used for regular Telecom or FBG inscribed fibers, while WDM is restricted to the latter. When speaking of spatial resolution, OFDR can provide extremely high spatial resolutions, as high as 10 microns [34], [35]. The downside is that it becomes hard to reach fast refresh rates. WDM can interrogate FBGs that typically have a minimum spacing of $10 \mathrm{~mm}$. The spatial resolution is thus worse, but the aquisition speed is much higher $(\approx 1 \mathrm{kHz})$, which is suitable for real-time applications. While OFDR is the preferred technique to achieve high spatial resolution, its higher commercial cost [36], typically low SNR [10] and lower wavelength measurement accuracy are disadvantages in comparison to WDM-based interrogators. 


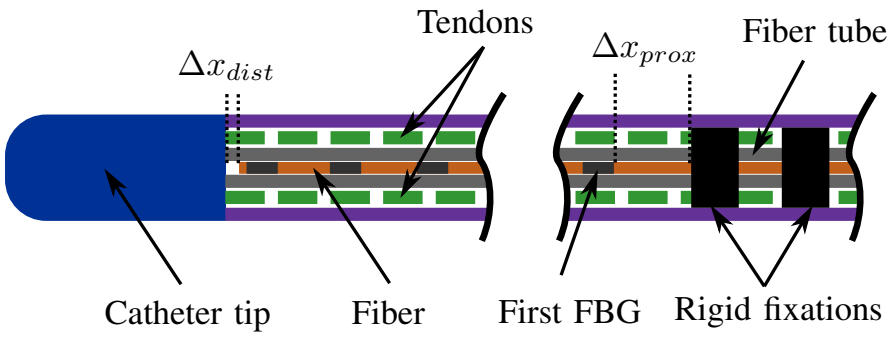

Fig. 2. Schematic illustrating the proposed method to integrate a fiber within a flexible structure, such as a catheter.

\section{B. Fiber Integration}

Figures 2, 3(a) and 3(b) show the proposed integration method of an MCF into a conventional catheter. For steerable catheters, normally one or more tendons are used to control the amount of bending of the catheter's tip. An inner working channel may or may not exist, depending on the catheter's desired function. The fiber's centerline is ideally coaxial with the catheter's centerline, but can be placed at an offset if the offset is known and constant throughout the length of the fiber. The offset can be accounted for in the reconstruction algorithm. The following design guidelines are proposed:

a) the spacing between the MCF and the inner lumen of the catheter must be reduced to a minimum. This can be done by incorporating a tube to fill in the extra space within the lumen. It is preferable to have the tube internally coated with a low-friction material to reduce traction as such may induce transverse stresses on the MCF.

b) a strong fixation, or preferably two, are made at the MCF's base. These can be made using adhesives such as epoxy, fiber ferrules, or other non-destructive methods. The most distal fixation from the base is used to hold the MCF in position, while the proximal fixation is used to prevent external forces from affecting the base grating. The base grating must be completely decoupled from external disturbances to maintain directionality and is therefore placed at a distance $\Delta x_{\text {prox }}$ from the most distal fixation point. $\Delta x_{\text {prox }}$ depends on the catheter/MCF configuration. In practice, a value $\geq 15$ $\mathrm{mm}$ was found to work well.

c) the MCF's base must be rigidly attached at a location where its tip is at an amount $\Delta x_{\text {dist }}$ from the edge. This extra space at the tip helps to prevent it from ever having contact. MCF tip contact has proven to significantly alter back reflections and disrupt wavelength measurements. Ideally $\Delta x_{\text {dist }}$ must be always $>0 \mathrm{~mm}$. A value larger than $1-2 \mathrm{~mm}$ was found to work well in practice.

\section{Twist Compensation}

Fiber twist, i.e. rotation of the fiber's material frame about the longitudinal axis, has always been a considerable issue in fiber-based shape sensing; regardless of the fiber configuration or interrogation method. The reason for this is the much lower sensitivity in sensing strain due to twist $\epsilon_{t}$, also known as torsional strain, even for considerable twist angles. Therefore, in the vast majority of implementations, the fiber is integrated in such a way as to prevent twist. Standard catheter bodies

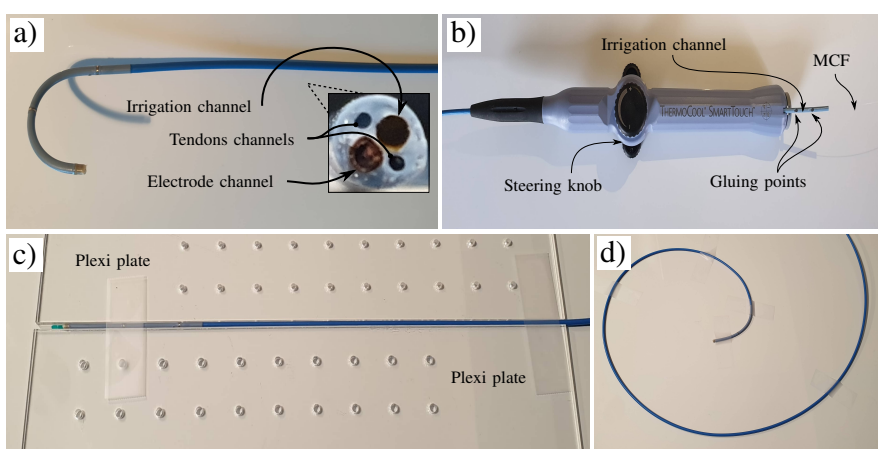

Fig. 3. a) catheter tip with MCF and FBGs inside, b) catheter handle with MCF inserted into the irrigation channel, c) step one of the calibration, d) step two of the calibration

exhibit a high torsional to bending stiffness ratio, which means that they can easily bend, but not twist. This, to a certain degree, solves the issue of twist during operation. In addition, a few recent developments have been proposed to measure twist, e.g. using twisted or helically wrapped fibers [37], [38]. These techniques offer promising solutions, but are still in their infancy and require further work in terms of characterization and optimization to be used in a practical setting.

Another crucial aspect is the MCF's internal twist; an important observation that was made during this work is that MCF fibers exhibit such intrinsic twist. Even if the fiber is in an externally unloaded state, or is placed on a plane, a nonnegligible amount of twist will cause - if not compensated for - the fiber to appear out-of-plane. In this situation, bend angles $\theta_{b}[n]$ are not constant, as would normally be expected. The way to compensate for this twist is discussed in the next section.

\section{Calibration for twist compensation}

A simple two-step process is proposed for calibration. Note that this method also holds for configurations with $N$ single core fibers, but will require further calibration steps to account for extra offsets and misalignments [11], [39]. The calibration process must be performed after the MCF has been integrated into the catheter. The two steps are illustrated in Fig. 3(c) and (d), and described in the following: 1) the interrogated length of the catheter, i.e. the part of the catheter between the location of the first FBG and the last FBG, is positioned in a straight line and with no externally induced forces. It is preferable to perform this step on a horizontal flat table top. FBG wavelengths are recorded, which will serve as the reference for the wavelengths $\left.\lambda_{0}[n] ; 2\right)$ the interrogated length of the catheter is placed on a flat surface. The catheter is bent such that the entire interrogated length is in-plane and under curvature, e.g. $\kappa=0.16 \mathrm{~cm}^{-1}$. The exact amount of bending/curvature is not relevant, and does not have to be constant. In an ideal case, there should be no twist (i.e. $\theta_{b}[n]=$ cte.). However, as previously explained, $\theta_{b}[n]$ is not constant in reality. The bending angles due to intrinsic twist $\theta_{b, t}[n]$ are therefore computed using (7) as outlined in Section II. Their value is constant and does not change during operation. The shape reconstruction algorithm is then modified to compensate 
for the contribution of the twist $\left(\theta_{b, t}[n]\right)$ onto the bend angle $\theta_{b}[n]$ as:

$$
\theta_{b, c o m p}[n]=\theta_{b}[n]-\theta_{b, t}[n],
$$

where the compensated bending angles $\theta_{b, c o m p}[n]$ are then used to compute the torsion $\tau[n]$, instead of $\theta_{b}[n]$. Note that $\theta_{b, t}[n]$ is stored and used throughout the lifespan of the catheter.

\section{E. Parameter Identification}

The shape reconstruction model relies on a variety of input parameters. An accurate identification of these parameters is necessary to obtain accurate shape estimation. It has been observed that slight deviations in the values of some of these parameters have conspicuous effects on the final estimation accuracy. The model's prominent input parameters are:

1) distance between consecutive FBGs, $l_{z}(\mathrm{~m})$

2) angular offset between consecutive cores, $\Delta \theta(\mathrm{rad})$

3) distance between an outer core and central core, $r(\mathrm{~m})$

4) strain sensitivity coefficient, $S_{\epsilon}$

Most MCF manufacturers report values for these parameters. However, these are typically averaged values and can differ from one MCF to another. Henken et al. have presented an elaborated paper regarding error analysis based on model input parameters of FBGs [40]. Furthermore, as an example, it has been observed that a two percent error in $S_{\epsilon}$ can have significant impact on the final shape accuracy.

The following procedure to have a good estimation of model parameters is proposed:

1) the catheter, with the embedded MCF, is positioned in a variety of 2D and 3D shapes with known ground truth. Ground truth can be obtained through various ways such as bi-plane imaging, electromagnetic (EM) tracking, 3D printed moulds with known shapes, etc. Hence, for each configuration, there will be a ground truth curve $C_{s, g r}(s)$.

2) an optimization problem is defined that minimizes the objective cost function $C(\Theta)$ :

$$
\boldsymbol{C}(\boldsymbol{\Theta})=\sum_{i=1}^{k} \max \left(\boldsymbol{d}\left(C_{s, g r}(s, k)-C_{s, r c}(s, k, \boldsymbol{\Theta})\right)\right),
$$

for $\Theta$ subject to

$$
\left\{\begin{array}{l}
\Theta=\left[\Delta \theta, r, S_{\epsilon}\right] \\
\Delta \theta_{\min } \leq \Delta \theta \leq \Delta \theta_{\max } \\
r_{\min } \leq r \leq r_{\max } \\
S_{\epsilon, \min } \leq S_{\epsilon} \leq S_{\epsilon, \max }
\end{array}\right.
$$

where $k$ is the index of the $k^{t h}$ configuration, $\Theta$ is the vector of the model input parameters to be optimized, $C_{s, r c}(s, k, \boldsymbol{\Theta})$ is the reconstructed curve based on the optimization parameters $\boldsymbol{\Theta}$, and $\boldsymbol{d}\left(C_{s, g r}(s, k)-C_{s, r c}(s, k, \boldsymbol{\Theta})\right)$ is the Euclidean distances vector between every point on $C_{s, g r}(s, k)$ to the closest point on $C_{s, r c}(s, k, \boldsymbol{\Theta})$. Note that the base frames of the ground truth curve $C_{s, g r}(s, k)$ and the reconstructed curve $C_{s, r c}(s, k, \boldsymbol{\Theta})$ are normally non-coincident. Hence, a rigid transformation using the Iterative Closest Point (ICP) algorithm followed by a brute-force translational perturbation are performed to align both curves [41].

The distance between consecutive FBGs $l_{z}$ was not considered in the optimization problem. The reason for this is because $l_{z}$ can be more easily controlled during fiber manufacturing, and its value is normally given precisely. While there exist many optimization algorithms, the trust-region reflective optimization algorithm [42] was employed for this problem.

\section{F. Reconstruction Algorithm}

Three modifications to the introduced reconstruction algorithm are proposed to improve shape accuracy. These are: 1) addition of twist compensation, 2) solving the differential Frenet-Serret equations and reconstructing the spatial curve (8) using the Helical Extension Method (HEM) [31], and 3) analysing different interpolation techniques and employing the one with the best performance. The first modification has already been elaborated previously.

Lim et al. [31] have proposed HEM as an alternative method to determine a three-dimensional space curve given its curvature and torsion profiles. While the conventional approach to solve the differential Frenet-Serret equations utilizes methods such as the crude Euler method and the $4^{\text {th }}$ order Runge-Kutta, HEM constructs a helical arc segment between consecutive points. The helix parameters such as radius and height are obtained from the curvature and torsion at that point. Lim et al. claim that their method has proven its enhanced performance and computational ease with respect to other conventional methods, e.g. Euler and Runge-kutta. Finally, four different interpolation techniques: cubic spline, piecewise cubic hermite interpolating polynomial (PCHIP), k-nearest neighbour, and linear were analysed for the interpolation of the discrete curvature $\kappa[n]$ and torsion $\tau[n]$ profiles.

\section{EXPERIMENTAL SETUP}

A seven-core MCF with an outer diameter of 200 microns from FBGS International (Geel, Belgium) was embedded into the irrigation channel of an $8 \mathrm{Fr}$ ablation catheter from BiosenseWebster (Irvine, CA, USA), and fixed into the channel using epoxy (see Fig. 3). Three adjacent outer cores with a core separation angle $\Delta \theta=\pi / 3$ were used. Note that expressions (4) and (5) are still valid for this configuration as they apply for general cases. The MCF contained 18 FBGs with distance $l_{z}=10 \mathrm{~mm}$, and was interrogated using the WDM-based FBG-Scan 804D interrogator from FBGS International (Geel, Belgium). The measurement rate was set at $100 \mathrm{~Hz}$. The fiber parameters reported by the manufacturer were $\boldsymbol{\Theta}_{\text {org }}=[1.047,3.75 e-5,0.777]$.

\section{A. Static tests}

A total of six 2D and six 3D shapes were prepared to obtain ground truth curves $C_{s, g r}(s, k)$ (see Fig. 4). Three shapes were used for parameter optimization and three for validation. The $2 \mathrm{D}$ shapes were obtained by creating grooves as mathematical functions within a plate. For 3D shapes, custom 3D printed tubes with predefined mathematical functions 

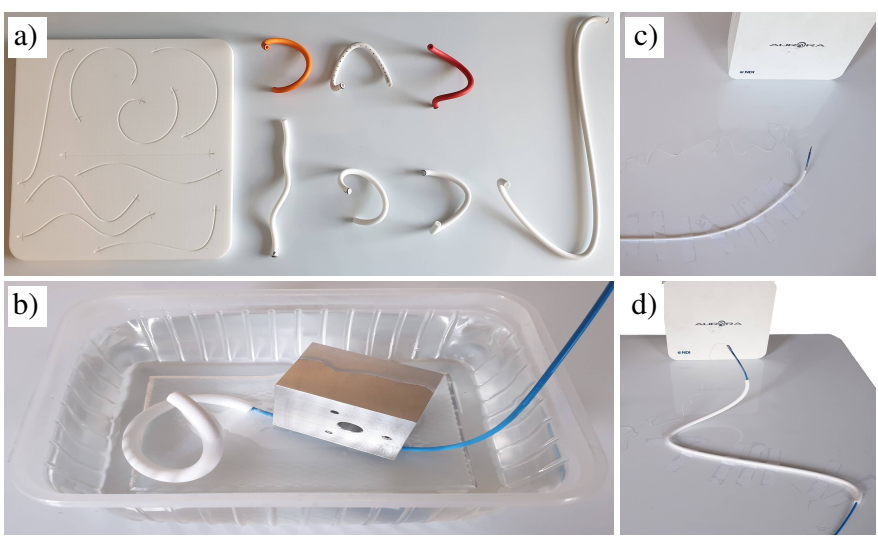

Fig. 4. a) plate with groves for $2 \mathrm{D}$ validation, $3 \mathrm{D}$ printed tubes for $3 \mathrm{D}$ validation, and a rigid 3D printed sheath, b) catheter inserted into a 3D printed tube and submerged into a hot water bath, c) catheter with EM sensor at the tip inserted into a pre-bent $8 \mathrm{Fr}$ sheath from BioSense Webster, d) catheter with EM sensor at the tip inserted into the rigid 3D printed sheath

were manufactured. The inner diameter of these tubes was $3 \mathrm{~mm}$. The arc lengths of the $2 \mathrm{D}$ and $3 \mathrm{D}$ shapes were all $170 \mathrm{~mm}$ long, which coincides with the interrogated length of the catheter. The mathematical curves used were chosen to exhibit diverse curvature and torsion profiles. Practically, the maximum curvature $\kappa$ and torsion $\tau$ values obtained were $0.675 \mathrm{~cm}^{-1}$ and $1.500 \mathrm{rad} \cdot \mathrm{cm}^{-1}$, respectively. Theoretically, the maximum curvature can be calculated using (3) and (4) and taking $\Delta \lambda_{B}$ as the maximum permissible wavelength shift.

The fiber was integrated into the catheter and calibrated using the methods proposed in Section III. For 2D tests, the interrogated length of the catheter was placed into the grooves of the plate. For 3D shapes, the catheter was inserted over the same length into the 3D printed tubes. For each configuration, the MCF wavelengths were recorded and used in the optimization, and $\max \left(\boldsymbol{d}\left(C_{s, g r}(s, k)-C_{s, r c}(s, k, \boldsymbol{\Theta})\right)\right)$ was computed. Experiments for $2 \mathrm{D}$ and $3 \mathrm{D}$ shapes were repeated five times per configuration, which yielded a total of 30 trials for optimization, and 30 trials for validation.

The effect of temperature variation was also investigated. This was done by submerging the catheter - which was embedded within a 3D printed shape - into a hot water bath after which it was left to cool down to room temperature (see Fig. 4(b)). The catheter tip position and temperature were monitored throughout the experiment.

\section{B. Dynamic tests}

Four tests were carried out to characterize the dynamic behaviour; namely, the effect of: 1) longitudinal catheter rotation, 2) repeatability, 3) tendon actuation, and 4) dynamic catheter movement were investigated. Catheter rotation was investigated by inserting the catheter into an $8 \mathrm{Fr}$ pre-bent BioSense Webster sheath, as shown in Fig. 4(c). An EM sensor was fixed to the catheter's tip. The catheter was then rotated and tip positions were measured for different rotation angles. Similarly, repeatability was investigated by inserting the catheter into and out of the same sheath. The catheter was always brought back to the same reference point, and variations in tip position were computed. The effect of tendon

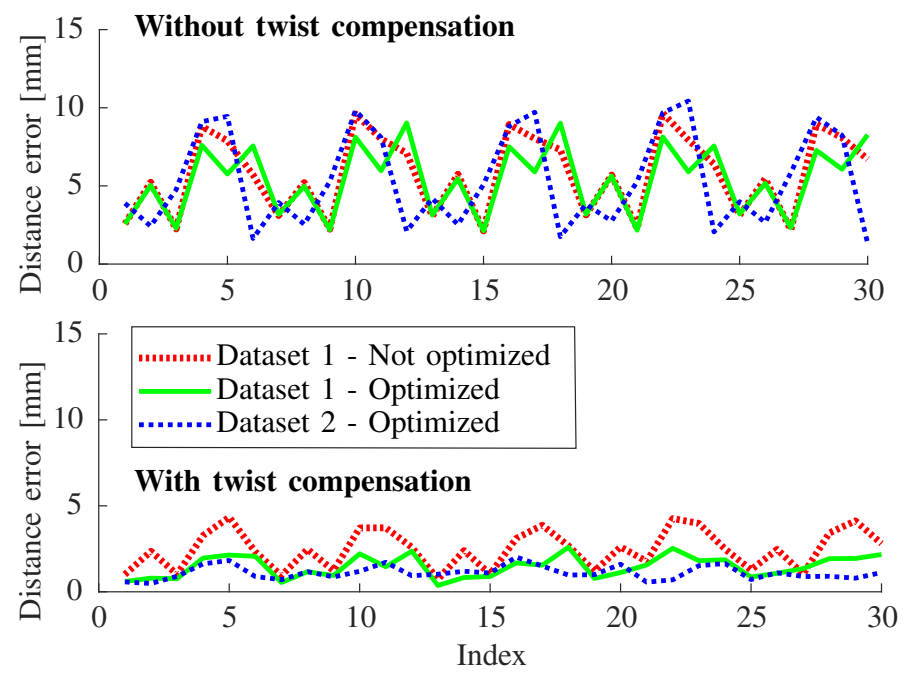

Fig. 5. Maximum distance errors between ground truth and reconstructed curves using original and optimized model parameters.

actuation was investigated by again inserting the catheter into the sheath. The flexible actuated part of the catheter was left protruding out of the sheath. An EM sensor was attached at the tip. The catheter tip was actuated back and forth several times using the steering knobs. The tip position computed from the shape reconstruction was compared to the tip position returned by the EM sensor data.

A $510 \mathrm{~mm}$ long PLA rigid tube with an inner diameter of $3 \mathrm{~mm}$ was 3D printed to resemble a sheath with known ground truth shape. The sheath was not internally lubricated, i.e. the inner surface was left rough. The effect of dynamic catheter movement was investigated by manually moving the catheter through the sheath. The reconstructed shape at given temporal intervals was compared with the shape of the sheath. Maximum distance errors were then computed. Data was gathered every $0.025 \mathrm{~s}$, but was down-sampled to $0.5 \mathrm{~s}$ intervals for post-processing. The catheter had an EM sensor attached to the tip to compute the speed of the tip.

\section{RESULTS}

\section{A. Static tests and algorithmic results}

1) Optimization, twist compensation and temperature: The optimized parameters were found to be $\boldsymbol{\Theta}_{\text {opt }}=[1.039,3.80 e$ $5,0.737]$. Results show a distinguishable variation in model parameters, especially for the strain sensitivity coefficient $S_{\epsilon}$ where the change was around $5.2 \%$. Figure 5 shows the comparative result for shape accuracy using original parameters versus optimized parameters, with and without twist compensation. First, it can be seen that twist compensation greatly improves accuracy, reducing the maximum error from $10.43 \mathrm{~mm}$ to $4.37 \mathrm{~mm}$ with mean errors of $2.33 \mathrm{~mm}$ and $0.85 \mathrm{~mm}$, respectively. Second, parameter optimization further improves the accuracy which yields a maximum error of 2.52 $\mathrm{mm}$ with a mean error of $0.70 \mathrm{~mm}$. Third, as can be seen in Fig. 5, it is clear that the optimized parameters also perform well for shapes excluded from the optimization (dataset 2). The clear improvement in accuracy signifies that proper calibration 

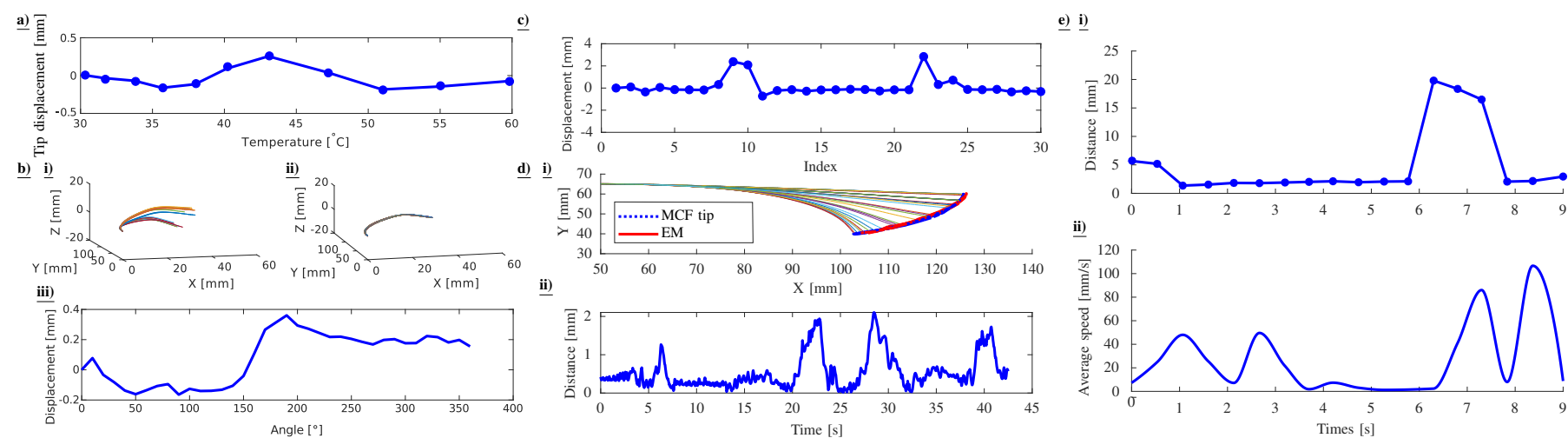

Fig. 6. a) tip displacement of a static catheter versus temperature, b) effect of catheter rotation along its longitudinal axis on tip position: i) shapes pre registration, ii) shape post registration, iii) tip displacement from a reference point for different rotation angles, c) displacement of the catheter's tip from a reference point for different trials, d) effect of tendons on reconstructed shape and comparison of tip position between MCF reconstruction and EM sensor, e) maximum distance error between the shapes of the moving catheter and 3D printed sheath

and twist compensation can indeed greatly improve shape accuracy.

Figure 6(a) shows the variation in catheter tip position magnitude versus temperature. The water bath temperature decreased from around $60^{\circ} \mathrm{C}$, which is higher than the average human body temperature, to $30^{\circ} \mathrm{C}$. Throughout this temperature decrease, the maximum position variation was $0.29 \mathrm{~mm}$. This indicates that external temperature effects are uniform throughout the fiber cores, and that it plays a negligible role in the final shape reconstruction.

2) HEM, interpolation and computational time: A comparison of the reconstructed curve has also been made using HEM and the conventional $4^{t h}$ order Runge-Kutta method. The maximum distance error between the two methods was comparable. However, the RMS of maximum distance errors dropped from $1.53 \mathrm{~mm}$ for Runge-Kutta to $1.40 \mathrm{~mm}$ for HEM $(\approx 8.5 \%$ drop). Using HEM for reconstruction, four different interpolation techniques were applied and compared: cubic spline, PCHIP, k-nearest neighbour, and linear. The maximum distance errors, in $\mathrm{mm}$, for these interpolation techniques were: $2.52,2.57,2.71$, and 2.82, respectively. While the differences are not large, it was found that cubic spline interpolation yields the best performance. The computational time for all interpolation methods averaged around $0.0143 \mathrm{~s}$ (maximum peaks were around $0.018 \mathrm{~s}$ ). There was no notable difference in computational time between the different interpolation methods. $c++$ code was used to run the reconstruction algorithm on a 64-bit operating system with $32 \mathrm{~GB}$ of RAM and a 2.60 $\mathrm{GHz}$ processor.

\section{B. Dynamic tests results}

1) Rotation and twist: Catheter rotation was investigated to observe the effect on shape accuracy and whether rotation induces further twist within the fiber. Figure 6(b, i) illustrates that the orientation of the shape reconstruction changes with angular rotation, which is a logical result. The TNB frame of the spatial curve is fixed while the bend on the catheter changes; hence the direction of the reconstructed curve should change. Figure $6(\mathrm{~b}$, ii) shows the different curves from different angular rotations. After registration, all the curves coincide and show a maximum tip deviation of $0.36 \mathrm{~mm}$. This is an important result as it indicates that external twist is negligible due to: $a$ ) the catheter's high torsional stiffness; even without lubrication which is normally present due to the patient's blood, and $b$ ) the freedom of the fiber within the catheter, such that twist of the catheter does not necessarily result in fiber twist.

2) Repeatability: As can be seen from Fig. 6(c), the catheter shape demonstrates excellent repeatability $\pm 0.82 \mathrm{~mm}$ (standard deviation) and a maximum variation of $2.84 \mathrm{~mm}$ was found. Since the experiment was carried out in a real medical sheath with a pre-bend, and without internal lubrication, the results are highly likely to be reproducible in real-life interventions.

3) Tendon actuation: Standard ablation catheters have separate channels for tendon actuation. Due to the isolation between the MCF and the tendons, it is expected that the catheter shape reconstruction caused by tendon deformation would be similar to the one from external deformation. This behaviour is confirmed in Fig. 6(d). The tip trajectory based on MCF shape reconstruction matches the one from the EM sensor with a maximum deviation of $2.1 \mathrm{~mm}$, which is lower than some of the highly curved static shape deformations. This indicates adequate shape reconstruction during tendon actuation, and that the tendons exert negligible pressure and strain on the fiber.

4) Catheter in sheath motion: Figure 6(e) depicts maximum distance errors between the dynamic catheter shape and the sheath. On average, the maximum distance error is $\approx 2.5$ $\mathrm{mm}$, which is similar to the static case. In cases where there are sharp bends and highly compressive forces, the distance errors can go as high as $21.2 \mathrm{~mm}$. These errors however, could be mitigated by applying lubrication within the sheath, and using an MCF with larger FBG wavelength spacing to allow for large curvatures and avoid wavelength peak overlap in the reflected spectrum.

\section{CONCLUSIONS}

In this paper, different factors that affect shape reconstruction were elaborated. It was shown that proper integration of the MCF within the catheter can significantly increase the estimation accuracy. Furthermore, compensation of intrinsic 
fiber twist has shown to significantly improve shape accuracy. Aspects such as proper calibration, parameter identification, and algorithm optimization have been discussed, and mitigating solutions provided. A characterization of the MCFembedded catheter in diverse quasi-realistic static and dynamic configurations was provided. The performance in all these situation was validated. For static shapes, mean and maximum spatial errors were $0.70 \mathrm{~mm}(0.41 \%)$ and $2.52 \mathrm{~mm}(1.48 \%)$ respectively, and similar for dynamic cases. Future work will focus on in-vivo testing.

\section{ACKNOWLEDGMENT}

The authors would like to thank the Flemish Agency of Innovation and Entrepreneurship (VLAIO) for funding this work (HBC.2018.2046), and the internal KU Leuven C2 project $S F-R E A C T A$.

\section{REFERENCES}

[1] S. M. Klute, et al., "Fiber-optic shape sensing and distributed strain measurements on a morphing chevron," in Collect. Tech. Pap. - 44th AIAA Aerosp. Sci. Meet., vol. 10, 2006, pp. 7460-7482.

[2] J. P. Moore, et al., "Photogrammetric verification of fiber optic shape sensors on flexible aerospace structures," in 2012 IEEE Avion. FiberOpt. Photonics Technol. Conf. AVFOP 2012, 2012, pp. 9-10.

[3] L. Zhang, et al., "On SDM/WDM FBG sensor net for shape detection of endoscope," in IEEE Int. Conf. Mechatronics Autom. ICMA 2005, vol. 4, no. July. IEEE, 2005, pp. 1986-1991.

[4] R. J. Roesthuis, et al., "Three-dimensional needle shape reconstruction using an array of fiber bragg grating sensors," IEEE/ASME Trans. Mechatronics, vol. 19, no. 4, pp. 1115-1126, 2014.

[5] S. Sefati, et al., "FBG-Based Control of a Continuum Manipulator Interacting with Obstacles," in IEEE Int. Conf. Intell. Robot. Syst., 2018, pp. 6477-6483.

[6] N. Lagakos, et al., "Microbend fiber-optic sensor," Appl. Opt., vol. 26, no. 11, pp. 2171--2180, 1987.

[7] M. Amanzadeh, et al., "Recent developments in fibre optic shape sensing," Meas. J. Int. Meas. Confed., vol. 128, pp. 119-137, 2018.

[8] C. Shi, et al., "Shape sensing techniques for continuum robots in minimally invasive surgery: A survey," IEEE Trans. Biomed. Eng., vol. 64, no. 8, pp. 1665-1678, 2017.

[9] F. Taffoni, et al., "Optical fiber-based MR-compatible sensors for medical applications: An overview," Sensors (Basel), vol. 13, no. 10, pp. 14 105-14 120, 2013.

[10] R. G. Duncan, et al., "High-accuracy fiber-optic shape sensing," in Sens. Syst. Networks Phenomena, Technol. Appl. NDE Heal. Monit. 2007, vol. 6530, 2007, p. 65301S.

[11] M. Abayazid, et al., "3D flexible needle steering in soft-tissue phantoms using Fiber Bragg Grating sensors," in Proc. - IEEE Int. Conf. Robot. Autom., 2013, pp. 5843-5849.

[12] K. Henken, et al., "Accuracy of needle position measurements using fiber Bragg gratings," Minim. Invasive Ther. Allied Technol., vol. 21, no. 6, pp. 408-414, 2012.

[13] N. J. van de Berg, et al., "Design of an actively controlled steerable needle with tendon actuation and FBG-based shape sensing," Med. Eng. Phys., vol. 37, no. 6, pp. 617-622, 2015.

[14] S. Sefati, et al., "FBG-Based Position Estimation of Highly Deformable Continuum Manipulators: Model-Dependent vs. Data-Driven Approaches," in 2019 Int. Symp. Med. Robot. ISMR 2019, 2019, pp. 1-6.

[15] B. Kim, et al., "Optimizing curvature sensor placement for fast, accurate shape sensing of continuum robots," in Proc. - IEEE Int. Conf. Robot. Autom., 2014, pp. 5374-5379.

[16] R. J. Roesthuis and S. Misra, "Steering of Multisegment Continuum Manipulators Using Rigid-Link Modeling and FBG-Based Shape Sensing," IEEE Trans. Robot., vol. 32, no. 2, pp. 372-382, 2016.

[17] S. C. Ryu and P. E. Dupont, "FBG-based shape sensing tubes for continuum robots," in Proc. - IEEE Int. Conf. Robot. Autom., 2014, pp. 3531-3537.
[18] R. J. Roesthuis, et al., "On using an array of fiber Bragg grating sensors for closed-loop control of flexible minimally invasive surgical instruments," in IEEE Int. Conf. Intell. Robot. Syst., 2013, pp. 25452551.

[19] S. Jäckle, et al., "Shape Sensing with Fiber Bragg Grating Sensors: A Realistic Model of Curvature Interpolation for Shape Reconstruction," in Inform. aktuell, 2019, pp. 258-263.

[20] H. Nijland, et al., "Evaluation of Accuracy and Precision of CT-guidance in Radiofrequency Ablation for Osteoid Osteoma in 86 Patients," PLoS One, vol. 12, no. 4, pp. 1-11, 2017.

[21] F. Bourier, et al., "Accuracy assessment of catheter guidance technology in electrophysiology procedures: A comparison of a new 3D-based fluoroscopy navigation system to current electroanatomic mapping systems," J. Cardiovasc. Electrophysiol., vol. 25, no. 1, pp. 74-83, 2014.

[22] F. Bourier, et al., "Sensor-Based Electromagnetic Navigation (Mediguide $\mathbb{R}$ ): How Accurate Is It? A Phantom Model Study," J. Cardiovasc. Electrophysiol., vol. 26, no. 10, pp. 1140-1145, 2015.

[23] X. Yi, et al., "An innovative 3D colonoscope shape sensing sensor based on FBG sensor array," in Proc. 2007 Int. Conf. Inf. Acquis. ICIA, 2007, pp. 227-232.

[24] S. Elayaperumal, et al., "Autonomous real-time interventional scan plane control with a 3-d shape-sensing needle," IEEE Trans. Med. Imaging, vol. 33, no. 11, pp. 2128-2139, 2014.

[25] M. J. Gander, et al., "Bend measurement using Bragg gratings in multicore fibre," Electron. Lett., vol. 36, no. 2, pp. 120-121, 2000.

[26] F. Parent, et al., "Enhancement of accuracy in shape sensing of surgical needles using optical frequency domain reflectometry in optical fibers," Biomed. Opt. Express, vol. 8, no. 4, pp. 2210-2221, 2017.

[27] P. Leyendecker and R. Haslinger, "Fiber optic curvature sensor," in Proc. IEEE Sensors, 2014, pp. 43-46.

[28] E. M. Lally, et al., "Fiber optic shape sensing for monitoring of flexible structures," in Sensors Smart Struct. Technol. Civil, Mech. Aerosp. Syst. 2012, vol. 8345, 2012, p. 83452Y.

[29] F. Khan, et al., "Multi-Core Optical Fibers with Bragg Gratings as Shape Sensor for Flexible Medical Instruments," IEEE Sens. J., vol. 19, no. 14, pp. 5878-5884, 2019.

[30] H. Wang, et al., "Shape Detection Algorithm for Soft Manipulator Based on Fiber Bragg Gratings," IEEE/ASME Trans. Mechatronics, vol. 21, no. 6, pp. 2977-2982, 2016.

[31] S. Lim and S. Han, "Helical extension method for solving the natural equation of a space curve," Surf. Topogr. Metrol. Prop., vol. 5, no. 3, pp. 1-8, 2017.

[32] J. P. Moore and M. D. Rogge, "Shape sensing using multi-core fiber optic cable and parametric curve solutions," Opt. Express, vol. 20, no. 3 , pp. 2967-2973, 2012.

[33] T. Banchoff and S. Lovett, Differential geometry of curves and surfaces. CRC Press, 2010.

[34] B. A. Childers, et al., "Use of 3000 Bragg grating strain sensors distributed on four eight-meter optical fibers during static load tests of a composite structure," in Smart Struct. Mater. 2001 Ind. Commer. Appl. Smart Struct. Technol., vol. 4332, 2001, pp. 133-142.

[35] K. T. V. Grattan and B. T. Meggitt, Optical Fiber Sensor Technology. Springer, Dordrecht, 1995.

[36] L. Schenato, "A review of distributed fibre optic sensors for geohydrological applications," Appl. Sci., vol. 7, no. 9, p. 896, 2017.

[37] R. Xu, et al., "Shape sensing for torsionally compliant concentric-tube robots," in Opt. Fibers Sensors Med. Diagnostics Treat. Appl. XVI, vol. 9702, 2016, p. 97020V.

[38] P. S. Westbrook, et al., "Continuous Multicore Optical Fiber Grating Arrays for Distributed Sensing Applications," J. Light. Technol., vol. 35, no. 6, pp. 1248-1252, 2017.

[39] S. Sefati, et al., "A highly sensitive fiber Bragg Grating shape sensor for continuum manipulators with large deflections," in Proc. IEEE Sensors, vol. 2017-Decem, 2017, pp. 1-3.

[40] K. R. Henken, et al., "Error analysis of FBG-based shape sensors for medical needle tracking," IEEE/ASME Trans. Mechatronics, vol. 19, no. 5, pp. 1523-1531, 2014.

[41] K. Niu, et al., "Feasibility of A-mode ultrasound based intraoperative registration in computer-aided orthopedic surgery: A simulation and experimental study," PLoS One, vol. 13, no. 6, pp. 1-15, 2018.

[42] T. M. Le, et al., "Numerical optimization applying trust-region reflective least squares algorithm with constraints to optimize the non-linear creep parameters of soft soil," Appl. Math. Model., vol. 41, pp. 236-256, 2017. 